\title{
A note on the flow of a homogeneous intrusion into a two-layer fluid
}

\author{
G. C. HOCKING ${ }^{1}$ and L. K. FORBES ${ }^{2}$ \\ ${ }^{1}$ Mathematics \& Statistics, Murdoch University, Murdoch, WA, 6150, Australia \\ email: G.Hocking@murdoch.edu.au \\ ${ }^{2}$ School of Mathematics \& Physics, University of Tasmania, Hobart, Australia
}

(Received 2 August 2007; revised 23 February 2007)

\begin{abstract}
The intrusion of a constant density fluid at the interface of a two-layer fluid is considered. Numerical solutions are computed for a model of a steady intrusion resulting from flow down a bank and across a broad lake or reservoir. The incoming fluid is homogeneous and spreads across the lake at its level of neutral buoyancy. Solutions are obtained for a range of different inflow angles, flow rate and density differences. Except in extreme cases, the nature of the solution is predicted quite well by linear theory, with the wavelength at any Froude number given by a dispersion relation and wave steepness determined largely by entry angle. However, some extreme solutions with rounded meandering flows and non-unique solutions in the parameter space are also obtained.
\end{abstract}

\section{Introduction}

The intrusion of a constant density fluid into a two-layer stratification, such as might occur when a riverine inflow enters a stratified reservoir, is considered. In two recent papers [3, 4], a periodic intrusion flow of this type was considered and it was found that there are two branches of solution. At higher speeds, the two interfaces were found to be in phase, whereas at slower speeds they were out of phase. This lower branch was found to spawn a branch of solitary waves in the long-wave limit.

Benjamin [1] considered a gravity current on the bottom of a channel and there are subsequent experimental works by Manins [7], Mehta et al. [8] and Sutherland et al. [11]. Flynn and Sutherland [2] considered the flow from the anvil of a thundercloud and showed that it may form an intrusion into the surrounding atmosphere. A field study was conducted by Hebbert et al. [6] for particular application to a river inflow to reservoirs, and field data confirming the existence of both bottom and mid-level intrusions was obtained. Parau and Dias [10] considered flows similar to these but with all three layers in motion. Nield and Woods [9] showed that such flows could also occur during volcanic eruptions.

The purpose of the work described herein is to extend the earlier results to examine the effect of the angle at which the water enters the lake. This is found to provide not only solutions predicted by the earlier work but also a separate branch of very rounded, meandering flows that exist in the same parameter space. Assuming a steady state for the intruding flow, a number of solutions can be computed that show that the wave steepness 


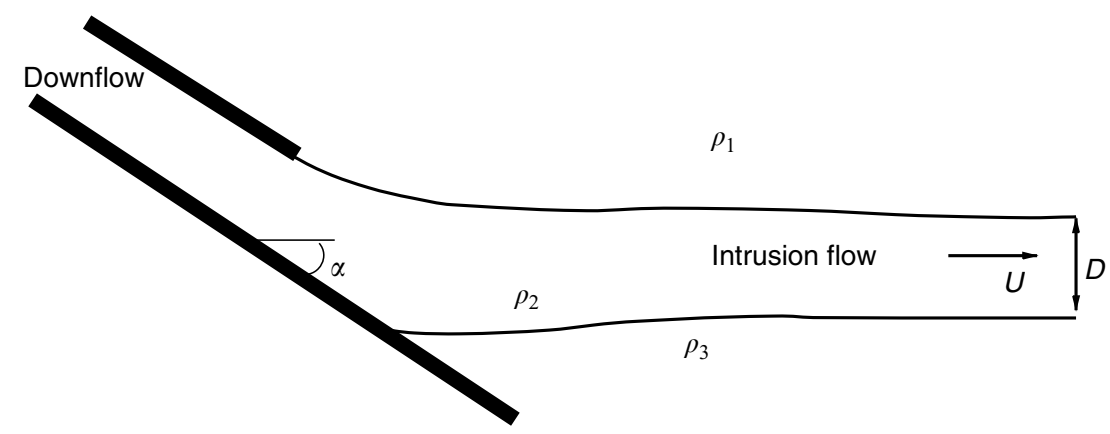

FIGURE 1. Definition sketch showing the horizontal intrusion breaking away from the reservoir bottom slope. Downstream horizontal velocity $U$ and depth $D$.

(amplitude divided by wavelength) of the waves on the intrusion depends on the angle of the bottom slope as the water begins its intrusion path, whereas the wavelength and Froude number are determined (for given density differences) by a dispersion relationship.

If the entry slope is zero (horizontal intrusion), there is a solution that is a purely horizontal flow with uniform velocity. As the angle increases, waves are shown to develop that increase in amplitude. In cases of lower flow rates, these steepen until reaching a critical height, and hence a limiting configuration with sharp crests and broad troughs. At higher flow rates, however, the intrusion becomes a narrow jet with a very steep rounded shape.

However, another group of solutions with very rounded waves is found that break down when they become unphysical in the sense that they curl over and intersect their own surface, as was found by Grundy and Tuck [5] for a similar problem in which a layer of air trailing a hovercraft was sitting on a free water surface. Furthermore, in the limit as entry angle approaches zero, these solutions do not become horizontal like the branch above. No attempt is made to resolve Kelvin-Helmholtz instabilities on the interface, although it is possible that many of the solutions computed in this paper may have some kind of shear instability.

Section 2 discusses the solution method, while subsequent sections describe the numerical method, results and give some conclusions.

\section{Problem formulation and solution method}

The steady motion of an inviscid, incompressible fluid that lies between two stagnant regions is investigated. The intruding fluid is assumed to be neutrally buoyant within the domain and to intrude horizontally between two constant density layers as shown in Figure 1. The density of the fluid above the intrusion is $\rho_{1}$, in the intrusion itself, $\rho_{2}$, and beneath the intrusion $\rho_{3}$. We define two parameters $\gamma_{1}=\rho_{1} / \rho_{2}$, and $\gamma_{2}=\rho_{3} / \rho_{2}$, and for a stable density configuration, we require $\gamma_{1}<1$ and $\gamma_{2}>1$. The method seeks a complex potential $f(z)=\phi+i \psi$, where the real and imaginary parts satisfy Laplace's equation $\nabla^{2} \phi=0$ and $\nabla^{2} \psi=0$ within the intrusion flow.

At this point, it will save us some work if we nondimensionalize with respect to the downstream intrusion velocity $U$ and the downstream intrusion layer thickness $D$. Along 
the top and bottom of the intrusion at $y=\eta_{U}(x), \eta_{L}(x)$, respectively, assuming the upper and lower layers to be stagnant, the Bernoulli equation indicates that the pressure should be constant along the interfaces, so that

$$
\begin{gathered}
\eta_{U}-1+\frac{F^{2}}{2\left(1-\gamma_{1}\right)}\left(u^{2}+v^{2}-1\right)=0 \quad \text { on } y=\eta_{U}(x), \\
\eta_{L}+\frac{F^{2}}{2\left(1-\gamma_{2}\right)}\left(u^{2}+v^{2}-1\right)=0 \quad \text { on } y=\eta_{L}(x) .
\end{gathered}
$$

Here, $F=U(g D)^{-1 / 2}$ is a Froude number representing the inflow velocity and $u$ and $v$ are horizontal and vertical components of velocity in the intrusion layer. In non-dimensional variables, the downstream intrusion velocity and thickness are both of unit value, and the parameters of the problem are $\gamma_{1}, \gamma_{2}, F$ and $\alpha$, where $\alpha$ is the angle of the downslope.

We choose to solve this problem by finding an analytic function, $\Omega=\theta+i \tau$, defined by the relation,

$$
f^{\prime}(z)=u-i v=\exp (-i \Omega(z))
$$

where $z=x+i y$ is the independent space variable in the complex plane. Therefore, $\theta$ is the angle that any streamline makes to the horizontal and $e^{\tau}$ is the speed of the fluid. In this formulation, $\Omega$ is an analytic function and hence we can apply Cauchy's integral formula around the boundary of the flow domain. On the downslope of the lake, the angle $\alpha$ is known.

To avoid the complications of the upstream inflow conditions, and since our interest is in the intrusion itself, we place a rigid lid on the top of the intruding fluid until it reaches the level of neutral buoyancy.

The method of solution is to transform the flow domain to the $f$-plane via the half-plane as shown in Figure 2, using the transformation $w=e^{\pi f}$, and noting that

$$
z(w)=z(1)+\frac{1}{\pi} \int_{1}^{w} \frac{e^{i \theta-\tau}}{w} d w
$$

where $z(1)=(0,0)$ without loss of generality, then

$$
\begin{aligned}
& x(w)=\frac{1}{\pi} \int_{1}^{w} \frac{e^{-\tau} \cos \theta}{w} d w, \\
& y(w)=\frac{1}{\pi} \int_{1}^{w} \frac{e^{-\tau} \sin \theta}{w} d w .
\end{aligned}
$$

These mappings allow us to apply Cauchy's integral formula to $\Omega$ in the $w$-plane from $\mathscr{R}\{w\} \in(-\infty, \infty)$, and we know from the boundary shape that $\theta=\alpha$ on the real axis, where $w_{T}<w<w_{B}=1$, while $\theta$ remains unknown on the remainder of the real axis. Noting that $\tau \rightarrow 0$ as $w \rightarrow \infty$, and taking the imaginary part of the contour integral, we find

$$
\tau\left(w_{0}\right)=-\alpha \ln \left(\frac{1-w_{0}}{w_{T}-w_{0}}\right)-\frac{1}{\pi} \int_{-\infty}^{w_{T}} \frac{\theta(w)}{w-w_{0}} d w-\frac{1}{\pi} \int_{1}^{\infty} \frac{\theta(w)}{w-w_{0}} d w
$$

This equation can be combined with the dynamic conditions (2.1) on the top and bottom 


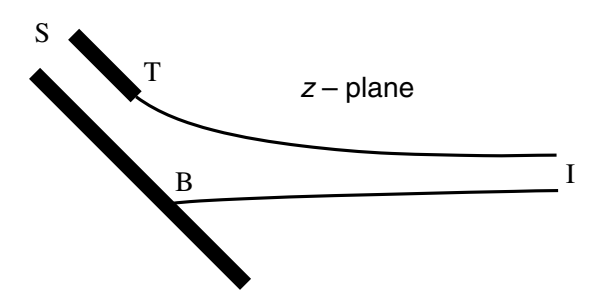

(a)

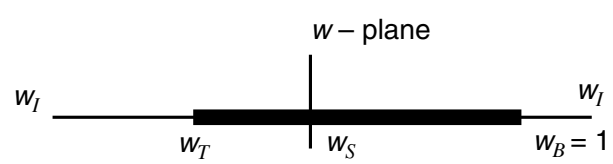

(b)



(c)

Figure 2. Mappings used in solution (a) $z$-plane, (b) $w$-plane and (c) $f$-plane.

of the intrusion layer, which in terms of these variables become

$$
\eta_{U}\left(w_{T}\right)+\frac{1}{\pi} \int_{w_{T}}^{w} e^{-\tau(\omega)} \sin \theta(\omega) \frac{d \omega}{\omega}+\frac{F^{2}}{2\left(1-\gamma_{1}\right)}\left[e^{2 \tau(w)}-1\right]=0
$$

on the upper surface $y=\eta_{U}(w),-\infty<w<w_{T}$, and

$$
\eta_{L}(1)+\frac{1}{\pi} \int_{1}^{w} e^{-\tau(\omega)} \sin \theta(\omega) \frac{d \omega}{\omega}-1+\frac{F^{2}}{2\left(1-\gamma_{1}\right)}\left[e^{2 \tau(w)}-1\right]=0
$$

on the lower surface $y=\eta_{L}(w), 1<w<\infty$. Differentiating and integrating both (2.8) and (2.9), it is possible to find an expression for $\tau(w)$ in each case as

$$
\tau(w)=\frac{1}{3} \ln \left[-\frac{3\left(1-\gamma_{1}\right)}{\pi F^{2}} \int_{w_{T}}^{w} \sin \theta(\omega) \frac{d \omega}{\omega}\right]
$$

on the upper surface $y=\eta_{U}(w),-\infty<w<w_{T}$, and

$$
\tau(w)=\frac{1}{3} \ln \left[e^{3 \tau_{T}}-\frac{3\left(1-\gamma_{2}\right)}{\pi F^{2}} \int_{1}^{w} \sin \theta(\omega) \frac{d \omega}{\omega}\right]
$$

on the lower surface $y=\eta_{L}(w), 1<w<\infty$.

The problem is to solve (2.7) with (2.10) and (2.11) for the unknown surface angle $\theta(w)$ on $-\infty<w<w_{T}$ and $1<w<\infty$, after which the interfaces can be obtained using (2.5) and (2.6). 


\section{Solution methods}

\subsection{A linear solution}

A linear solution assuming small displacement of the interfaces was computed by Forbes and colleagues $[3,4]$. In the variables used in this paper, the solution for the potential function can be written

$$
\phi(x, y)=[A(k) \cosh k y+B(k) \sinh k y] \sin (k x+\epsilon),
$$

where $B(k)=k A(k)\left(\left(F^{2} /\left(1-\gamma_{2}\right)\right), k\right.$ is the wavenumber and $F$ and $k$ are related by the dispersion relation

$$
F^{2}=\frac{\gamma_{2}-\gamma_{1}}{2 k \tanh k}\left[1 \pm\left(1+\frac{4\left(1-\gamma_{1}\right)\left(1-\gamma_{2}\right) \tanh ^{2} k}{\left(\gamma_{2}-\gamma_{1}\right)^{2}}\right)^{1 / 2}\right]
$$

where $0<y<1,0 \leqslant x<\infty$, and $\epsilon$ is the phase. In the earlier work, $\epsilon$ was set to zero without loss of generality. Here, we choose $\epsilon$ to satisfy the condition that the flow exits smoothly from the downflow. This choice is not unique, and so we make the minimum energy choice, i.e. that the steepest angle achieved by the waves is at the separation point, giving

$$
\begin{gathered}
\eta_{U}(x)=1-\frac{k F^{2}}{\left(1-\gamma_{1}\right)}(A(k) \cosh k+B(k) \sinh k) \cos (k x+\epsilon) \text { on } y=1, \\
\eta_{L}(x)=-\frac{k F^{2}}{\left(1-\gamma_{2}\right)} A(k) \cos (k x+\epsilon) \text { on } y=0
\end{gathered}
$$

for $0 \leqslant x<\infty$, where $\epsilon=\pi / 2$ and the waves are then in phase. The minimum energy argument provides an estimate for the amplitude, $\Lambda$, of the waves generated, as

$$
\Lambda=\frac{\tan (\alpha)}{k} \text {. }
$$

This value can be used to determine the wave steepness

$$
s=\frac{\Lambda}{\lambda}=\frac{\tan (\alpha)}{2 \pi} .
$$

This argument can only be applied directly for the case of equal density differences between the layers, since for different density differences the slope of the upper and lower interfaces at any point will not match in general. However, we might expect it to provide a guide in these cases as well.

\subsection{Numerical solution}

A full analytic solution of this problem is not available because of the nonlinear nature of the equations. However, numerical solution of such systems of integral equations is a relatively straightforward procedure, and provided one is careful, can be performed using a discretisation of the equations and numerical evaluation of the integrals using a trapezoidal scheme.

The method is to truncate the integration domain at large positive and negative values of $w$ as appropriate and then seek $\theta_{i}$ at a set of points along $w$, in particular along 
$1<w<\infty$ equating (2.7) and (2.11) and along $-\infty<w<w_{T}$ by equating (2.7) and (2.10). The integral (2.7) should be interpreted in the Cauchy-Principal value sense, and hence can be rendered nonsingular by adding and subtracting $\theta$ at $w=w_{0}$, so that

$$
\int_{A}^{B} \frac{\theta(w)-\theta\left(w_{0}\right)}{w-w_{0}} d w+\theta\left(w_{0}\right) \ln \left(\frac{B-w_{0}}{A-w_{0}}\right),
$$

where $A$ and $B$ are the ends of the appropriate integration domain, and noting that

$$
\lim _{w \rightarrow w_{0}} \frac{\theta(w)-\theta\left(w_{0}\right)}{w-w_{0}}=\theta^{\prime}\left(w_{0}\right) .
$$

The integrals are all now well behaved and can be accurately computed using the trapezoidal rule. The result is a set of $N$ nonlinear equations in the $N$ unknown surface angles and these equations can be solved using a damped Newton-Raphson iteration scheme. When convergence occurred, it was quite rapid, but in some cases it depended on the truncation point; if the truncation points did not fall at the crest or trough of the waves generated, then the method would not converge very well. Some experimentation however provided a full range of solutions. Values of $N$ up to 1,400 were used, and truncation points varied depending on the Froude number, but in most cases were chosen to resolve 4 or 5 wavelengths of the flow. Solutions were found to be accurate to graphical accuracy with $N=600$, i.e. with 300 points on each of the upper and lower interfaces.

\section{Simulations and results}

Two sets of simulations were performed. Firstly, the density difference between the intruding layer and the fluid above and below was assumed to be identical, leading to symmetric flow conditions on the two interfaces. In the second series, the density differences at the two interfaces were varied so that the resulting conditions were no longer symmetric.

\subsection{Symmetric density difference}

A series of simulations was performed for a range of different Froude numbers and entry angles of the stream. In this case, we used $\gamma_{1}=0.95$ and $\gamma_{2}=1.05$, and note that for this symmetric case the Froude number $F$ could be replaced by a densimetric Froude number $F_{D}^{2}=F^{2} /\left(1-\gamma_{1}\right)$, so there are only two parameters of importance in this case, i.e. $F_{D}$ and $\alpha$. However, for consistency the original parameterisation is retained.

At each Froude number, the inflow angle was gradually increased from zero $(\alpha$ decreased) and a succession of solutions was computed. It was found that at low values of Froude number, there was an upper limit on the entry angle for which solutions could be obtained. Although it is not absolutely clear from the solutions, it appears that the limiting configuration contains a limiting wave profile with a $120^{\circ}$ angle at the crest. Figure 3 shows two solutions for the case $F=0.282$, at angles $\alpha=-0.1$ and $\alpha=-0.4$.

At higher Froude numbers, it was found that solutions could be obtained right up to an entry angle of $\alpha=-\pi / 2$, i.e. vertical entry of the intrusion. In these solutions, the waves 




FIGURE 3. Intrusion shapes for $F=0.282$, with entry angle $\alpha=-0.1$ (solid) and $\alpha=-0.4$ (dashed), $\gamma_{1}=0.95, \gamma_{2}=1.05$.

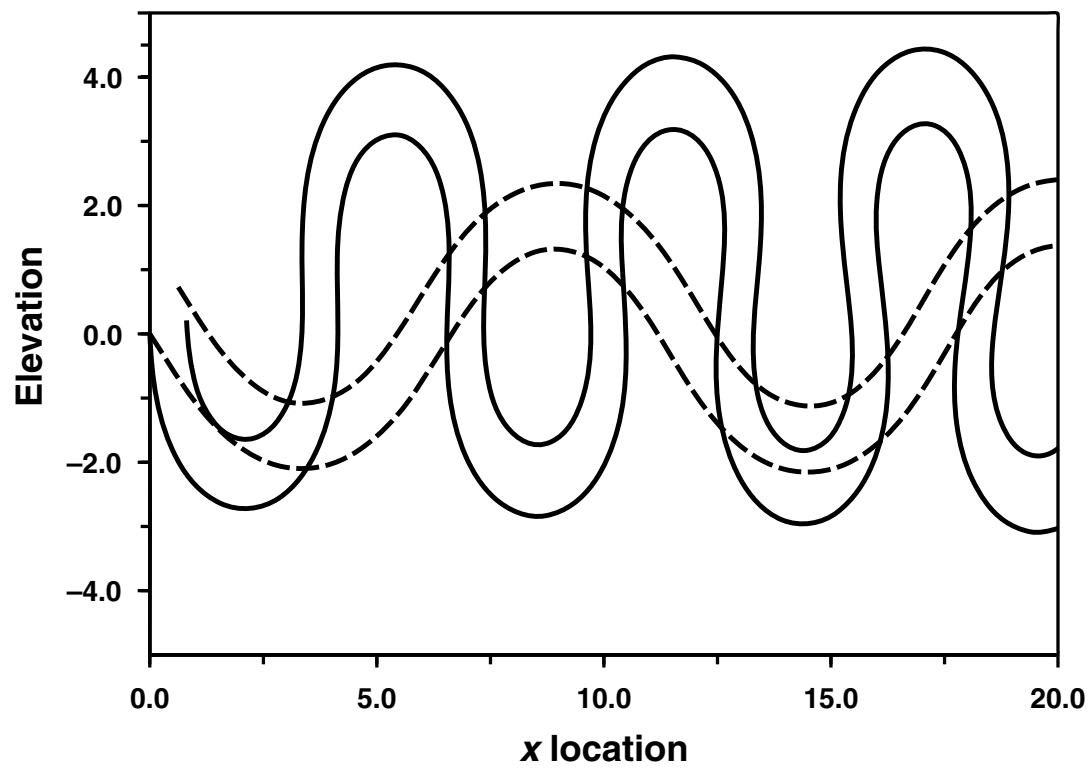

FIGURE 4. Intrusion shapes for $F=0.846$, with entry angle $\alpha=-0.8(--)$ and $\alpha=-1.54(-)$, $\gamma_{1}=0.95, \gamma_{2}=1.05$.

began to overhang at close to $\alpha=-\pi / 2$, although at no stage did successive waves come close to intersecting each other. Figure 4 shows solutions of this type, with $F=0.846$ for $\alpha=-0.8$ and $\alpha=-1.54$, close to vertical intrusion. 




FiguRE 5. Wave steepness for different entry angle at $F=0.564(-\times-), F=1.128(-\circ-)$, and estimated from equation (3.5) $(-), \gamma_{1}=0.95, \gamma_{2}=1.05$.

Overhanging waves of this type were previously found by Grundy and Tuck [5] while considering a similar problem of a jet of air from beneath a hovering vehicle above a free water surface. This is not surprising, since in that case both upper and lower layers are also stagnant. The differing densities can be accounted for by a different scaling of gravity.

In all cases, the waves on the two interfaces seemed to be in phase. For small to medium entry angles, the wavelength was accurately predicted by the dispersion relation (3.2). As Froude number increased, the wavelength increased in such a way that for a fixed entry angle the wave steepness ( $s=$ amplitude/wavelength) remained approximately constant, as can be seen in Figure 5, which shows the steepness against entry angle for $F=0.564$ and $F=1.124$ compared with the linearised calculation above. Clearly, the steepness is predictable for lower entry angles via equation (3.5).

However, the wave steepness begins to increase rapidly when entry angle is greater than about $70^{\circ}$, i.e. $\alpha \approx-1.2$, when the waves become rounded. As wave amplitude increases, the "waves" begin to look more like meandering streams than internal waves on a homogeneous layer of fluid. The waves appear to maintain a normal structure until close to limiting steepness, at which point instead of "breaking", their wavelength decreases quite rapidly and their amplitude grows, creating rounded, meandering waves.

The range of these solutions is given in Figure 6, and this is one of the main results of this work. It is clear that if the entry angle is fixed and Froude number decreases, there is a minimum Froude number beneath which no solutions of the type discussed here appear 


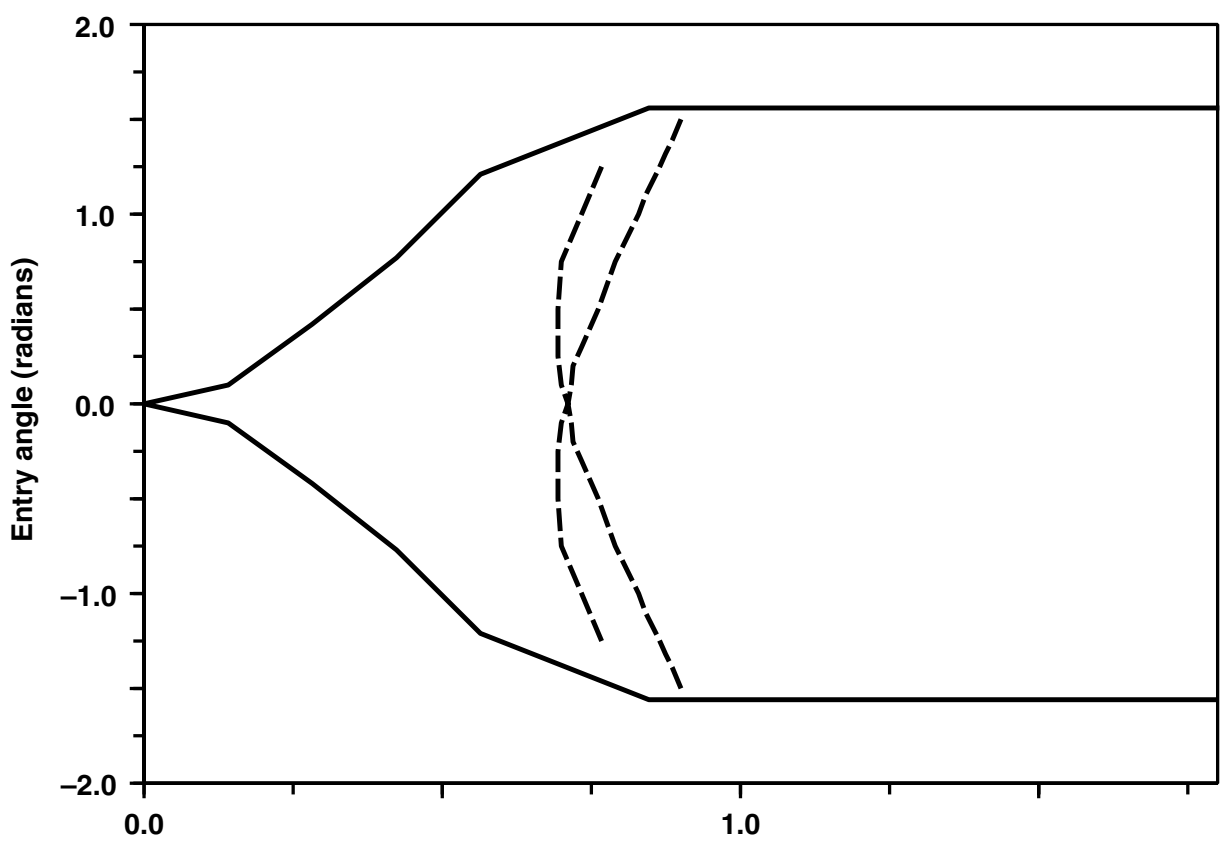

Froude number

Figure 6. Bounds on solutions of the two types for the cases $\gamma_{1}=0.95$ and $\gamma_{2}=1.05$. Solid lines are lower limits (in $F$ ) of normal solutions, whereas dashed lines represent lower limits (in $F$ ) of overhanging wave branches. Positive values of $\alpha$ indicate upward entry.

to exist. As the Froude number is increased, at fixed angle there does not appear to be a maximum and the wavelength just increases seemingly without bound. Positive values of $\alpha$ were computed out of academic interest, but might represent an upward flow at entry, such as an atmospheric intrusion of rising air off the side of a mountain.

All of the solutions discussed above appear to "grow" from the purely horizontal intrusion with no waves. However, starting the numerical scheme with random initial values of Froude number and entry angle gave a second branch of solutions for which the waves did not disappear as entry angle diminished. These solutions had a very rounded appearance for all entry angles similar to those found by Grundy and Tuck [5]. Limiting solutions were obtained when the overhanging portions of successive waves came into contact. An example of such a solution is shown for a case with zero entry angle (see Figure 7). It is interesting that a matching branch, vertically reflected in the horizontal axis, also exists. The lower limits of these two branches are shown in Figure 6 (as dashed lines), and it can be seen to overlap the previous branch, giving non-unique solutions under the same conditions. It is possible that one or more of these branches is an unstable steady state, and hence would not be observed. The term stability is used here with respect to perturbations of the order of the wavelength, rather than in the Kelvin-Helmholtz sense.

An interesting feature of the behaviour of these extra branches is that as the Froude number increases, they eventually join with the normal branch, and thus as $F$ decreases 


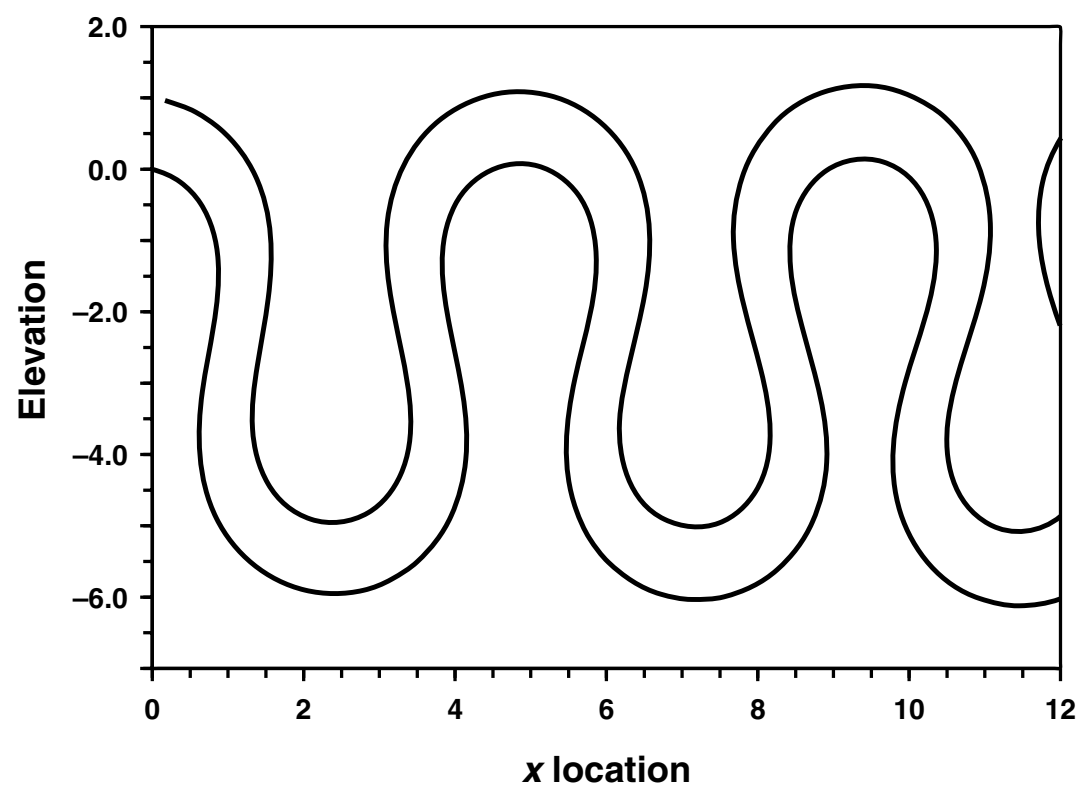

FigURE 7. Solution from curly branch for $\alpha=0, F=1.3, \gamma_{1}=0.95$ and $\gamma_{2}=1.05$. Break down occurs when successive waves intersect.

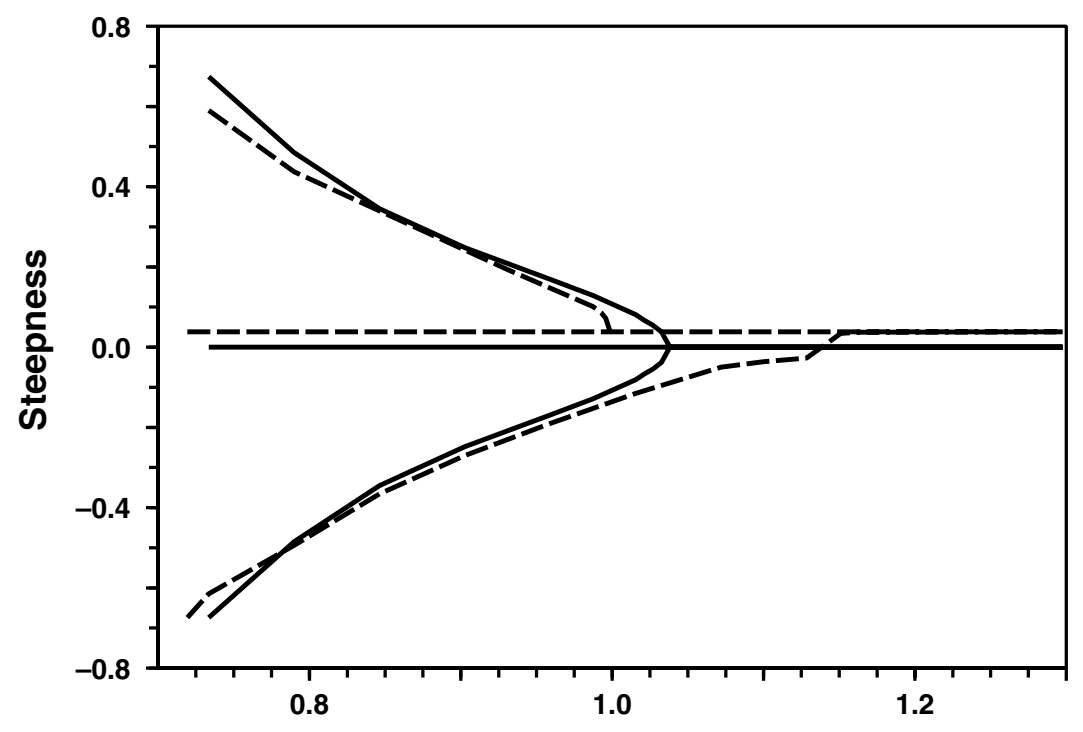

Froude number

FIGURE 8. Bifurcation of ordinary waves into the three branches as Froude number decreases for entry angle $\alpha=0(-)$ and $\alpha=-0.2(--)$.

at a given entry angle, there is a bifurcation in the solutions. Figure 8 shows this quite clearly for the two cases (i) $\alpha=0$, in which the behaviour is symmetric about zero wave steepness (shown as solid lines), and (ii) $\alpha=-0.2$, in which the separation from the 


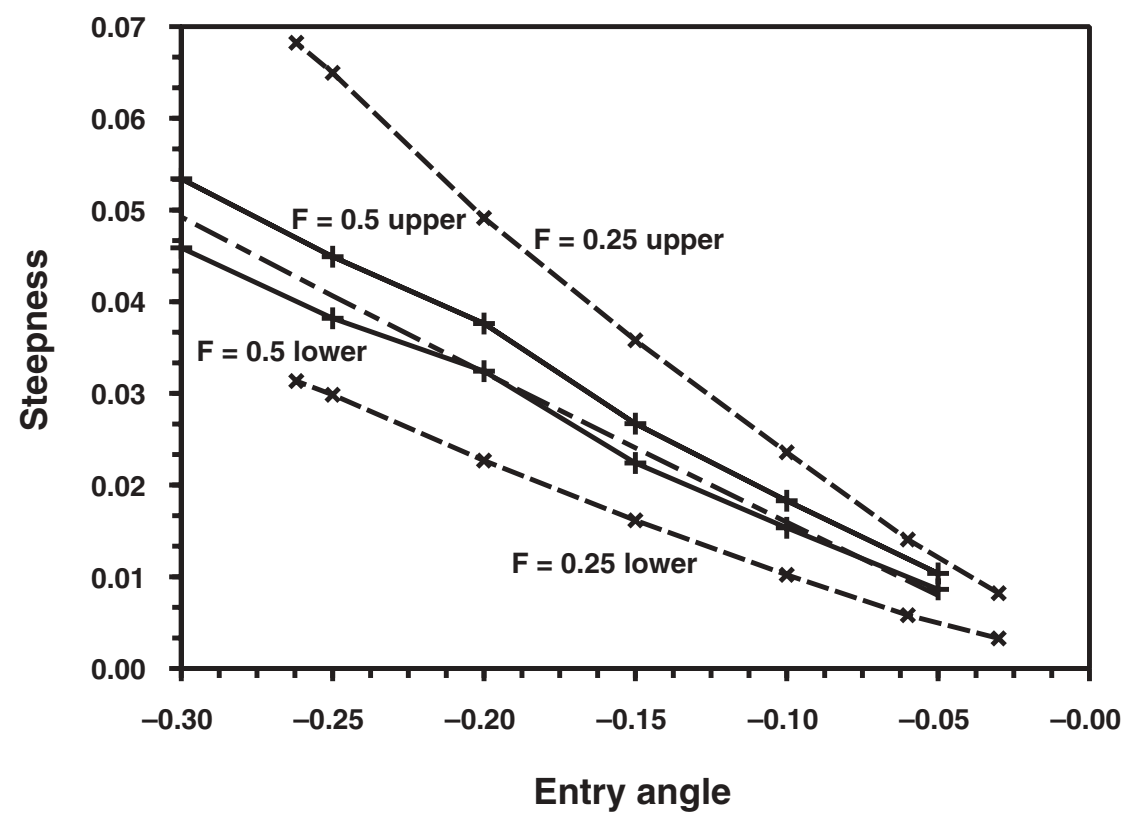

FIGURE 9. Wave steepness for the case $\gamma_{1}=0.9$ and $\gamma_{2}=1.01$, for $F=0.25$ and $F=0.5$ against that predicted by (3.5), the central dashed line.

normal wave branch occurs at different locations for the negative (in which most of the wave amplitude is below the entry point) and positive steepness (in which most is above).

\subsection{Non-symmetric density difference}

Simulations were carried out in the same manner as those above but with density differences that were no longer symmetric between the layers. The results were qualitatively very similar to the symmetric density difference case, and were not noticeable for small changes. However, as the differences became larger, more noticeable asymmetries in behaviour became apparent.

There are again several branches of solution, including the curly waves and the steepening sharp waves. At low Froude numbers, the limiting wave is a nonlinear wave with broad troughs and a sharp crest that approaches a stagnation point as the angle of entry is increased. At higher Froude numbers, the solutions become rounded such as those described in the previous section, and solutions look very similar to those in the symmetric case.

Again, the linearised solution and dispersion relation adequately describe the wavelength for different Froude numbers, and as above, the entry angle determines the wave amplitude. However, the latter is not quite so clear cut since the different density differences lead to different entry angles for the two interfaces (in the linear solution). It turns out that at low Froude numbers, the predicted wave steepness, (3.5), seems to be an average value for the upper and lower wave steepnesses, but as the Froude number increases, the steepness appears to be determined by this formula applied to the interface with the smaller density difference (the other steepness can then be determined from (3.2,3.3)). Figure 9 shows this clearly for the extremely asymmetric density case of $\gamma_{1}=0.9$ and $\gamma_{2}=1.01$. 




FIGURE 10. Interface shapes near the critical value for $F=0.25, \gamma_{1}=0.9$ and $\gamma_{2}=1.01$.

At $F=0.25$, the upper and lower interface wave steepnesses are equally spaced around the linear prediction, but for $F=0.5$, the lower interface has the smaller amplitude waves and their steepness is predicted quite well by (3.5). Note that the curve for $F=0.25$ terminates at $\alpha \approx-0.262$, and this is because a corner has begun to form on the upper interface at the wave crests. The interfaces for this case are shown in Figure 10, where it is clear that the peaks of the upper surface are sharpening and the troughs broadening as the limiting wave profile forms.

\section{Conclusions}

A numerical method has been used to solve model equations for a single layer, steady intrusion into a stagnant two-layer fluid. Solutions have been computed for a range of inflow angles, Froude numbers and density differences. It was found that for the symmetric density difference case the steepness of waves remains the same for a particular inflow angle, independent of the Froude number, and that this steepness can exceed the highest value $(s \approx 0.14)$ for an air-water interface in deep water (see, for example, Figure 7 in which $s \approx O(1)$ ). In these cases, however, the layers are so thin that they are no longer waves on an interface but a meandering jet in a stagnant two-layer fluid, and so this result is not violating the conclusions of earlier research.

Except in extreme cases, these solutions are quite well predicted using linear theory and a minimum energy argument. Further to this, there appears to be a nonuniqueness in the solutions, with solutions with standard wave appearance and with rounded meandering waves appearing at the same inflow angle and Froude number. It is likely that one of these two is unstable to perturbations of the order of the wavelength (rather than in the Kelvin-Helmholtz sense) and that such a steady state would not exist in practice, but it is 
beyond the scope of this work to resolve this issue. As the entry angle increases, the waves steepen, but at higher Froude number, instead of forming the limiting wave with a $120^{\circ}$ corner at the crest, the "wave" becomes rounded at the top (and bottom), thus rapidly increasing steepness beyond the usual limiting value. At these Froude numbers, however, the flow is no longer wave like but appears more like a meandering stream, with a thin jet of uniform density fluid speeding through a stagnant, two layer zone. For this particular geometry, forcing the inflow to begin in a particular way, no solutions of the out-of-phase type (at low values of $F$ ), as described by Forbes and Hocking [3] were obtained. It it possible for solutions with a stagnation point separation from the bottom surface to exist, and it would seem likely that these may be of this type, but despite attempts no such solutions were found. The combination of the results from this paper with the two earlier ones [3, 4] suggests that there may be a large degree of nonuniqueness in the solutions to the problem of a jet of constant density fluid intermediate to two stagnant layers, and that the form that is taken by the flow is importantly determined by the manner of its creation.

\section{References}

[1] Benjamin, T. B. (1968) Gravity Currents and related phenomena. J. Fluid Mech. 31, 209-248.

[2] Flynn, M. R. \& Sutherland, B. R. (2004) Intrusive gravity currents and internal gravity wave generation in stratified fluid. J. Fluid Mech. 514, 327-353.

[3] Forbes, L. K., Hocking, G. C. \& Farrow, D. E. (2006) An intrusion layer in stationary incompressible fluid: Part 1: Period waves. Eur. J. Appl. Maths 17, 557-575.

[4] Forbes, L. K. \& Hocking, G. C. (2007) An intrusion layer in stationary incompressible fluid: Part 2: A solitary wave. Eur. J. Appl. Maths 17, 577-595.

[5] Grundy, I. H. \& Tuck, E. O. (1987) Waves on a static water surface beneath a layer of moving air. J. Fluid. Mech. 178, 441-457.

[6] Hebbert, R., Imberger, J., Loh, I. \& Patterson, J. (1979) Collie River flow into the Wellington Reservoir. J. Hydr. Div., ASCE 105, 533-545.

[7] Manins, P. C. (1976) Intrusion into a stratified fluid. J. Fluid. Mech. 74, 547-560.

[8] Mehta, A. P., Sutherland, B. R. \& Kyba, P. J. (2002) Interfacial gravity waves II Wave excitation. Phys. Fluids 14, 3558-3569.

[9] Nield, S. E. \& Woods, A. W. (2004) Effects of flow density on the dynamics of dilute pyroclastic density currents. J. Volcanol. Geotherm. Res. 132, 269-281.

[10] Parau, E. \& Dias, F. (2001) Interfacial periodic waves of permanent form with free-surface boundary conditions. J. Fluid Mech. 437, 325-336.

[11] Sutherland, B. R., Kyba, P. J. \& Flynn, M. R. (2004) Intrusive gravity currents in two-layer fluids. J. Fluid Mech. 514, 327-353. 